\title{
Blowup or no blowup? The interplay between theory and numerics
}

\author{
Thomas Y. Hou ${ }^{\mathrm{a}, *}$, Ruo Li ${ }^{\mathrm{b}}$ \\ a Applied and Computational Math, 217-50, Caltech, Pasadena, CA 91125, USA \\ ${ }^{\mathrm{b}}$ LMAM\&School of Mathematical Sciences, Peking University, Beijing 100871, China
}

Available online 25 January 2008

\begin{abstract}
The question of whether the 3D incompressible Euler equations can develop a finite time singularity from smooth initial data has been an outstanding open problem in fluid dynamics and mathematics. Recent studies indicate that the local geometric regularity of vortex lines can lead to dynamic depletion of vortex stretching. Guided by the local non-blowup theory, we have performed large scale computations of the 3D Euler equations on some of the most promising blowup candidates. Our results show that there is tremendous dynamic depletion of vortex stretching. The local geometric regularity of vortex lines and the anisotropic solution structure play an important role in depleting the nonlinearity dynamically and thus prevents a finite time blowup.
\end{abstract}

(c) 2008 Elsevier B.V. All rights reserved.

PACS: 47.32.C-; 47.11.Kb

Keywords: Finite time singularities; 3D Euler equations; Spectral methods

\section{Introduction}

The question of whether the 3D incompressible Euler equations can develop a finite time singularity from smooth initial data is one of the most outstanding open problems in fluid dynamics and mathematics. This open problem is closely related to the Clay Millennium Open Problem on the 3D Navier-Stokes equations. The understanding of this problem could improve our understanding on the onset of turbulence and the intermittency properties of turbulent flows. A main difficulty in answering this question is the presence of vortex stretching, which gives a formal quadratic nonlinearity in vorticity. There have been many computational efforts in searching for finite time singularities of the 3D Euler equations, see e.g. [2-5,11-13,17,18,21-23]. For a more comprehensive review of this subject, we refer the reader to the book by Majda and Bertozzi [20] and the excellent review article by J. Gibbon in this issue [10].

Computing Euler singularities numerically is an extremely challenging task. First of all, it requires huge computational

\footnotetext{
* Corresponding author. Tel.: +1 626395 4546; fax: +1 6265780124 .

E-mail address: hou@ acm.caltech.edu (T.Y. Hou).
}

resources. Tremendous resolutions are required to capture the nearly singular behavior of the Euler equations. Secondly, one has to perform a careful convergence study. It is dangerous to interpret the blowup of an under-resolved computation as an evidence of finite time singularities for the 3D Euler equations. Thirdly, if we believe that the numerical solution we compute leads to a finite time blowup, we need to demonstrate the validation of the asymptotic blowup rate, i.e. is the blowup rate $\|\omega\|_{L^{\infty}} \approx \frac{C}{(T-t)^{\alpha}}$ asymptotically valid as $t \rightarrow T$ ? One also needs to check if the blowup rate of the numerical solution is consistent with the Beale-Kato-Majda non-blowup criterion [1] and other non-blowup criteria [7-9]. The interplay between theory and numerics is clearly essential in our search for Euler singularities.

There has been some interesting development in the theoretical understanding of the 3D incompressible Euler equations. It has been shown that the local geometric regularity of vortex lines can play an important role in depleting nonlinear vortex stretching [6-9]. In particular, the recent results obtained by Deng, Hou, and $\mathrm{Yu}[8,9]$ show that geometric regularity of vortex lines, even in an extremely localized region containing the maximum vorticity, can lead to depletion of nonlinear vortex stretching, thus avoiding finite 
time singularity formation of the 3D Euler equations. To obtain these results, Deng-Hou-Yu [8,9] explore the connection between the stretching of local vortex lines and the growth of vorticity. In particular, they show that if the vortex lines near the region of maximum vorticity satisfy some local geometric regularity conditions and the maximum velocity field is integrable in time, then no finite time blowup is possible. These localized non-blowup criteria provide stronger constraints on the local geometry of a potential finite time singularity. They can be used to re-examine some of the wellknown numerical evidences for finite time singularities of the 3D Euler equations.

\section{A brief review}

We begin with a brief review on the subject. Due to the formal quadratic nonlinearity in vortex stretching, only short time existence is known for the 3D Euler equations. One of the most well-known results on the 3D Euler equations is due to Beale-Kato-Majda [1] who show that the solution of the 3D Euler equations blows up at $T^{*}$ if and only if $\int_{0}^{T^{*}}\|\omega\|_{\infty}(t) \mathrm{d} t=$ $\infty$, where $\omega$ is vorticity.

There have been some interesting recent theoretical developments. In particular, Constantin-Fefferman-Majda [7] show that local geometric regularity of the unit vorticity vector can lead to depletion of the vortex stretching. Let $\xi=\omega /|\omega|$ be the unit vorticity vector and $\mathbf{u}$ be the velocity field. Roughly speaking, Constantin-Fefferman-Majda show that if (1) $\|\mathbf{u}\|_{\infty}$ is bounded in a $O(1)$ region containing the maximum vorticity. (2) $\int_{0}^{t}\|\nabla \xi\|_{\infty}^{2} \mathrm{~d} \tau$ is uniformly bounded for $t<T$, then the solution of the 3D Euler equations remains regular up to $t=T$.

There have been some numerical evidences which suggest a finite time blowup of the 3D Euler equations. One of the most well-known examples is the finite time collapse of two antiparallel vortex tubes by Kerr [17,18]. In Kerr's computations, he used a pseudo-spectral discretization in the $x$ and $y$ directions, and a Chebyshev discretization in the $z$ direction with resolution of order $512 \times 256 \times 192$. His computations showed that the maximum vorticity blows up like $O\left((T-t)^{-1}\right)$ with $T=18.9$. In his subsequent paper [18], Kerr showed that the maximum velocity blows up like $O\left((T-t)^{-1 / 2}\right)$ with $T$ being revised to $T=18.7$. It is worth noting that there is still a considerable gap between the predicted singularity time $T=$ 18.7 and the final time $t=17$ of Kerr's computations which he used as the primary evidence for the finite time singularity.

Kerr's blowup scenario is consistent with the Beale-KatoMajda non-blowup criterion [1] and the Constantin-FeffermanMajda non-blowup criterion [7]. But it falls into the critical case of the Deng-Hou-Yu local non-blowup criteria [8,9]. Below we describe the local non-blowup criteria of Deng-Hou-Yu.

\section{The local non-blowup criteria of Deng-Hou-Yu $[8,9]$}

Motivated by the result of [7], Deng, Hou, and Yu [8] have obtained a sharper non-blowup condition which uses only very localized information of the vortex lines. Assume that at each time $t$ there exists some vortex line segment $L_{t}$ on which the local maximum vorticity is comparable to the global maximum vorticity. Further, we denote $L(t)$ as the arclength of $L_{t}, \mathbf{n}$ the unit normal vector of $L_{t}$, and $\kappa$ the curvature of $L_{t}$.

Theorem 1 (Deng-Hou-Yu [8], 2005). Assume that (1) $\max _{L_{t}}(|\mathbf{u} \cdot \boldsymbol{\xi}|+|\mathbf{u} \cdot \mathbf{n}|) \leq C_{U}(T-t)^{-A}$ with $A<1$, and (2) $C_{L}(T-t)^{B} \leq L(t) \leq C_{0} / \max _{L_{t}}(|\kappa|,|\nabla \cdot \xi|)$ for $0 \leq t<T$. Then the solution of the $3 D$ Euler equations remains regular up to $t=T$ if $A+B<1$.

In Kerr's computations, the first condition of Theorem 1 is satisfied with $A=1 / 2$ if we use $\|\mathbf{u}\|_{\infty} \leq C(T-t)^{-1 / 2}$ as alleged in [18]. Kerr's computations suggested that $\kappa$ and $\nabla \cdot \xi$ are bounded by $O\left((T-t)^{-1 / 2}\right)$ in the inner region of size $(T-t)^{1 / 2} \times(T-t)^{1 / 2} \times(T-t)$ [18]. Moreover, the length of the vortex tube in the inner region is of order $(T-t)^{1 / 2}$. If we choose a vortex line segment of length $(T-t)^{1 / 2}$ (i.e. $B=1 / 2$ ), then the second condition is satisfied. However, we violate the condition $A+B<1$. Thus Kerr's computations fall into the critical case of Theorem 1. In a subsequent paper [9], Deng-Hou-Yu improved the non-blowup condition to include the critical case, $A+B=1$.

Theorem 2 (Deng-Hou-Yu [9], 2006). Under the same assumptions as Theorem 1, in the case of $A+B=1$, the solution of the $3 D$ Euler equations remains regular up to $t=T$ if the scaling constants $C_{U}, C_{L}$ and $C_{0}$ satisfy an algebraic inequality, $f\left(C_{U}, C_{L}, C_{0}\right)>0$.

We remark that this algebraic inequality can be checked numerically if we obtain a good estimate of these scaling constants. For example, if $C_{0}=0.1$, which seems reasonable since the vortex lines are relatively straight in the inner region, Theorem 2 would imply no blowup up to $T$ if $2 C_{U}<0.43 C_{L}$. Unfortunately, there was no estimate available for these scaling constants in [17]. One of our original motivations to repeat Kerr's computations using higher resolutions was to obtain a good estimate for these scaling constants.

\section{The high resolution 3D Euler computations of Hou and Li $[14,15]$}

In [14,15], we repeat Kerr's computations using two pseudospectral methods. The first pseudo-spectral method uses the standard $2 / 3$ dealiasing rule to remove the aliasing error. For the second pseudo-spectral method, we use a novel 36th order Fourier smoothing to remove the aliasing error. For the Fourier smoothing method, we use a Fourier smoother along the $x_{j}$ direction as follows: $\rho\left(2 k_{j} / N_{j}\right) \equiv \exp \left(-36\left(2 k_{j} / N_{j}\right)^{36}\right)$, where $k_{j}$ is the wave number $\left(\left|k_{j}\right| \leq N_{j} / 2\right)$. The time integration is performed by using the classical fourth order Runge-Kutta scheme. Adaptive time stepping is used to satisfy the CFL stability condition with CFL number equal to $\pi / 4$. In order to perform a careful resolution study, we use a sequence of resolutions: $768 \times 512 \times 1536,1024 \times 768 \times 2048$ and $1536 \times 1024 \times 3072$ in our computations. We compute the solution up to $t=19$, beyond the alleged singularity time $T=$ 18.7 by Kerr [18]. Our computations were performed using 
256 parallel processors with maximal memory consumption $120 \mathrm{~Gb}$. The largest number of grid points is close to 5 billions.

As a first step, we demonstrate that the two pseudo-spectral methods can be used to compute a singular solution arbitrarily close to the singularity time. For this purpose, we perform a careful convergence study of the two pseudo-spectral methods in both physical and spectral spaces for the 1D inviscid Burgers equation. The advantage of using the inviscid 1D Burgers equation is that it shares some essential difficulties as the 3D Euler equations, yet we have a semi-analytic formulation for its solution. By using the Newton iterative method, we can obtain an approximate solution to the exact solution up to 13 digits of accuracy. Moreover, we know exactly when a shock singularity will form in time. This enables us to perform a careful convergence study in both the physical space and the spectral space very close to the singularity time.

We have performed a sequence of resolution study with the largest resolution being $N=16,384$ [15]. Our extensive numerical results demonstrate that the pseudo-spectral method with the high order Fourier smoothing (the Fourier smoothing method for short) gives a much more accurate approximation than the pseudo-spectral method with the $2 / 3$ dealiasing rule (the $2 / 3$ dealiasing method for short). One of the interesting observations is that the unfiltered high frequency coefficients in the Fourier smoothing method approximate accurately the corresponding exact Fourier coefficients. Moreover, we observe that the Fourier smoothing method captures about $12 \sim$ $15 \%$ more effective Fourier modes than the $2 / 3$ dealiasing method in each dimension, see Fig. 1. The gain is even higher for the 3D Euler equations since the number of effective modes in the Fourier smoothing method is higher in three dimensions. Further, we find that the error produced by the Fourier smoothing method is highly localized near the region where the solution is most singular. In fact, the pointwise error decays exponentially fast away from the location of the shock singularities. On the other hand, the error produced by the $2 / 3$ dealiasing method spreads out to the entire domain as we approach the singularity time, see Fig. 2.

Next, we present our high resolution computations for the two anti-parallel vortex tubes [14]. We used the same initial condition whose analytic formula was given by Kerr (see Section III of [17], and also [14] for corrections of some typos in the description of the initial condition in [17]). However, there is some difference between our discretization and Kerr's discretization. We used a pseudo-spectral discretization in all three directions, while Kerr used a pseudo-spectral discretization only in the $x$ and $y$ directions and used a Chebyshev discretization in the $z$ direction. Based on the results of early tests, positive vorticity in the symmetry plane was imposed in the initial condition of Kerr [17]. How this was imposed as the vorticity field was mapped onto the Chebyshev mesh was not documented by Kerr [17]. This has led to some ambiguity in reproducing that initial condition which is being resolved by Kerr's group (private communication).

We first illustrate the dynamic evolution of the vortex tubes. In Figs. 4 and 5, we plot the isosurface of the 3D vortex tubes at $t=0$ and $t=6$ respectively. As we can see, the two initial

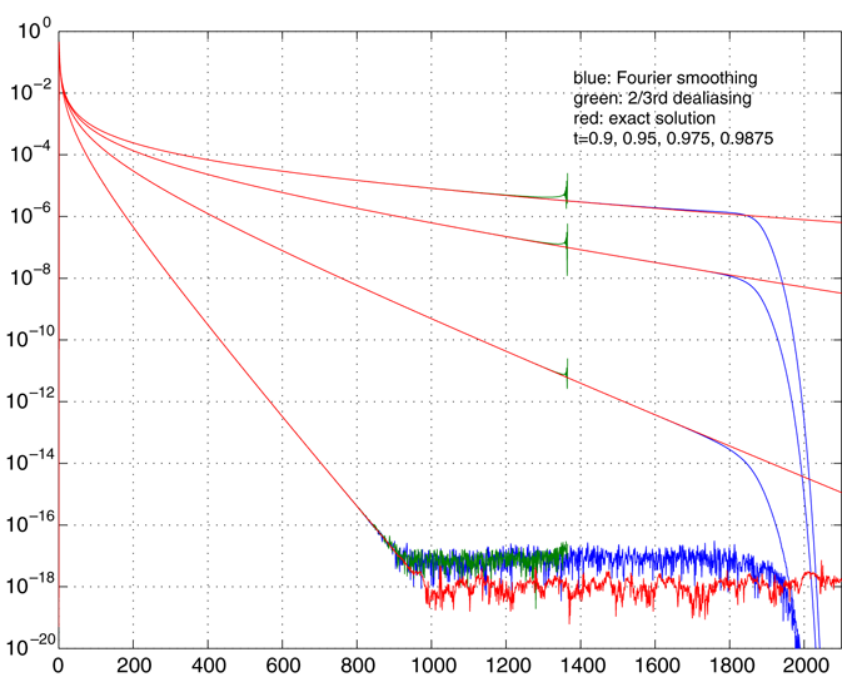

Fig. 1. Spectra comparison on different resolutions at a sequence of moments. The additional modes that kept the Fourier smoothing method higher than the $2 / 3$ rd dealiasing method are in fact correct. The initial condition is $u_{0}(x)=$ $\sin (x)$. The singularity time for this initial condition is $T=1$.

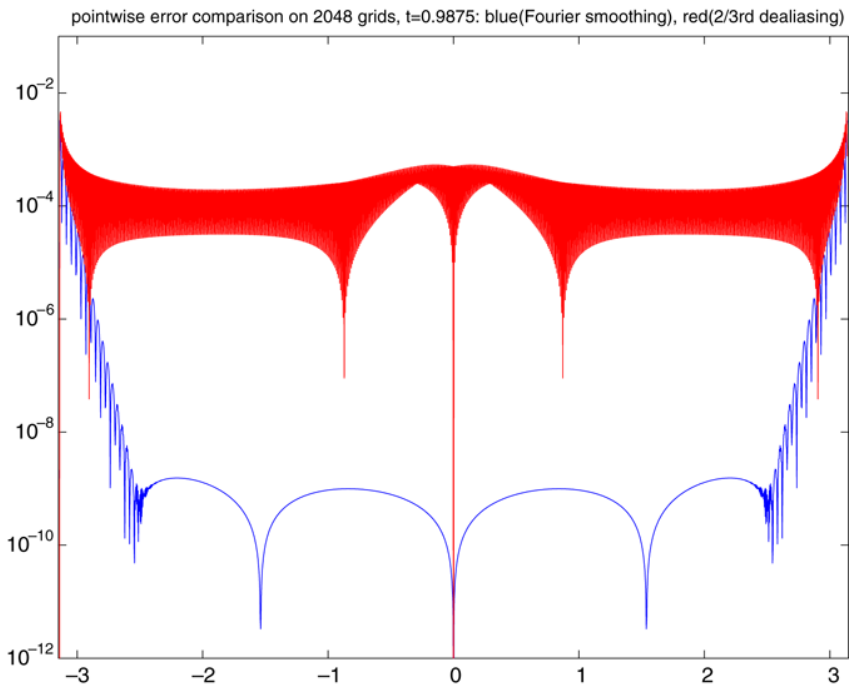

Fig. 2. Pointwise errors of the two pseudo-spectral methods as functions of time using different resolutions. The plot is in a log scale. The error of the $2 / 3 \mathrm{rd}$ dealiasing method (the top curve) is highly oscillatory and spreads out over the entire domain, while the error of the Fourier smoothing method (the bottom curve) is highly localized near the location of the shock singularity.

vortex tubes are very smooth and relatively symmetric. Due to the mutual attraction of the two anti-parallel vortex tubes, the two vortex tubes approach each other and become flattened dynamically. By time $t=6$, there is already a significant flattening near the center of the tubes. In Fig. 6, we plot the local 3D vortex structure of the upper vortex tube at $t=17$. By this time, the 3D vortex tube has essentially turned into a thin vortex sheet with rapidly decreasing thickness. The vortex lines become relatively straight. The vortex sheet rolls up near the left edge of the sheet.

We would like to make a few important observations. First of all, the maximum vorticity at later stage of the computation is actually located near the rolled-up region of the vortex sheet 


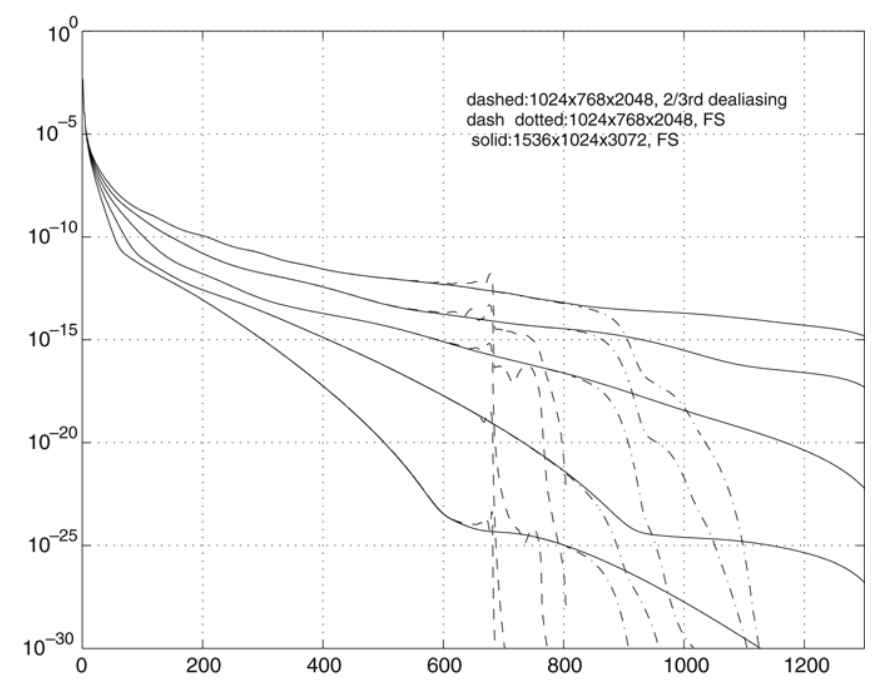

Fig. 3. The energy spectra vs wave numbers. The dashed lines and dash-dotted lines are the energy spectra with the resolution $1024 \times 768 \times 2048$ using the $2 / 3$ dealiasing rule and the Fourier smoothing, respectively. The times for the spectra lines are at $t=15,16,17,18,19$ respectively.

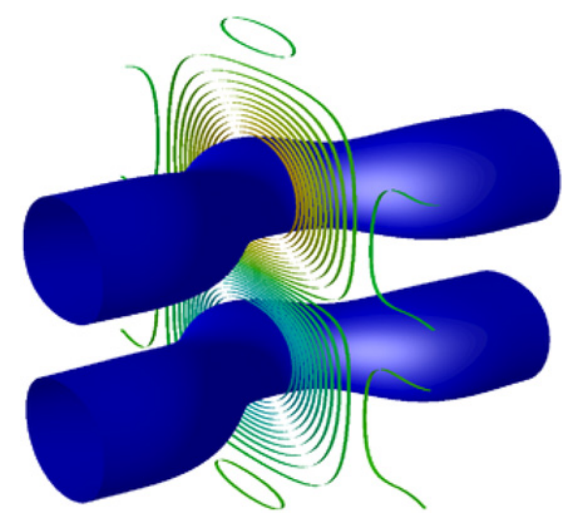

Fig. 4. The 3D view of the vortex tube at $t=0$.

and moves away from the bottom of the vortex sheet. Thus the mechanism of strong compression between the two vortex tubes becomes weaker dynamically at later time. Secondly, the location of maximum strain and that of maximum vorticity separate as time increases. Thirdly, the relatively "strong" growth of the maximum velocity between $t=15$ and $t=17$ becomes saturated after $t=17$ when the location of maximum vorticity moves to the rolled-up region, see Fig. 7. All these factors contribute to the dynamic depletion of vortex stretching. The origin of this behavior need to be analyzed in the future study.

We have performed a convergence study for the two numerical methods using a sequence of resolutions. For the Fourier smoothing method, we use the resolutions $768 \times 512 \times$ $1536,1024 \times 768 \times 2048$, and $1536 \times 1024 \times 3072$ respectively. Except for the computation on the largest resolution $1536 \times$ $1024 \times 3072$, all computations are carried out from $t=0$ to $t=$ 19. The computation on the final resolution $1536 \times 1024 \times 3072$ is started from $t=10$ with the initial condition given by the computation with the resolution $1024 \times 768 \times 2048$. For the $2 / 3$ dealiasing method, we use the resolutions $512 \times 384 \times 1024$,

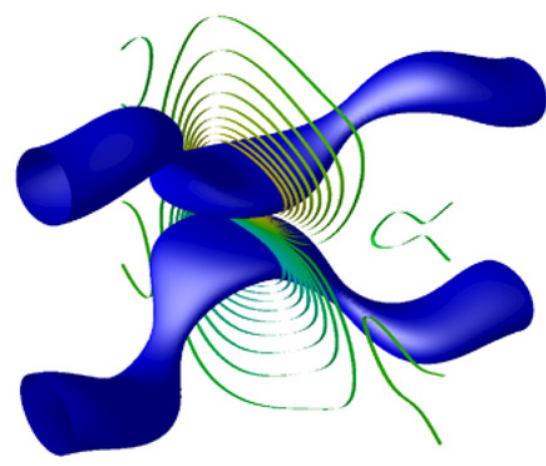

Fig. 5. The 3D view of the vortex tube at $t=6$.

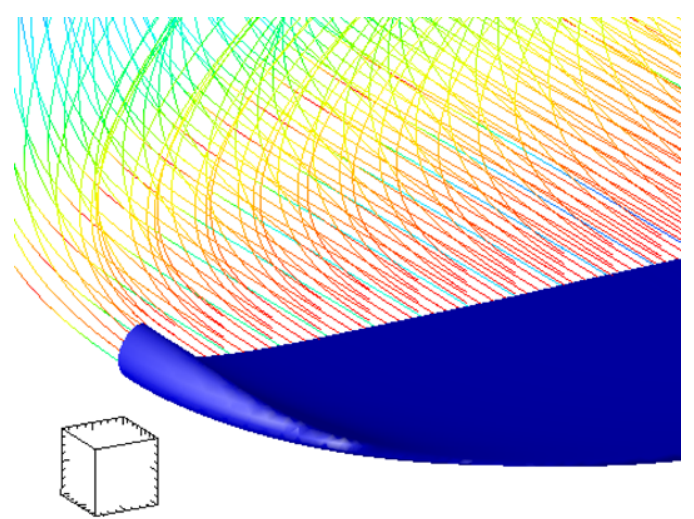

Fig. 6. The local 3D vortex structures and vortex lines around the maximum vorticity at $t=17$.

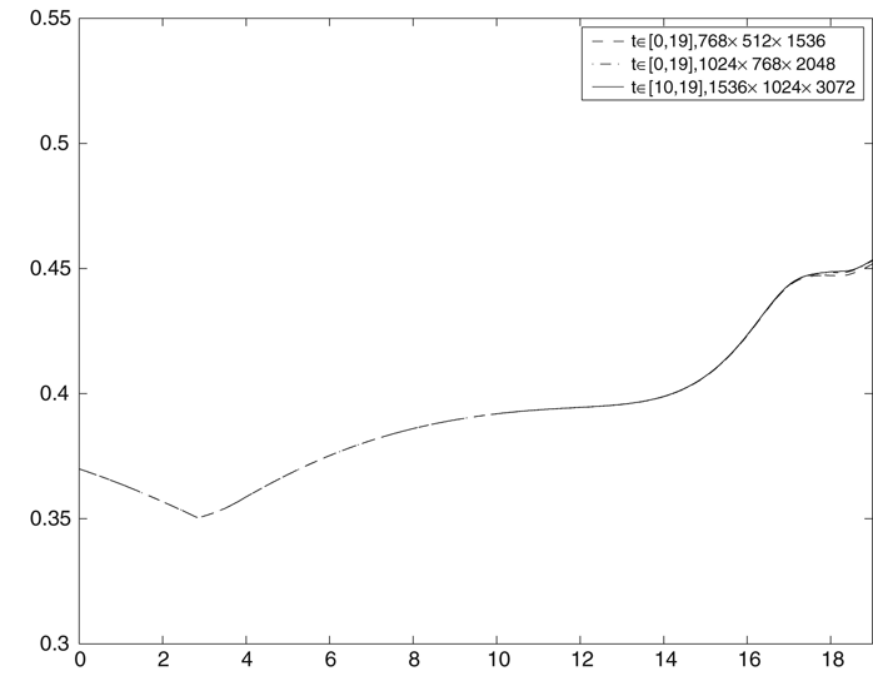

Fig. 7. Maximum velocity $\|\mathbf{u}\|_{\infty}$ in time using three different resolutions.

$768 \times 512 \times 1536$ and $1024 \times 768 \times 2048$ respectively. The computations using these three resolutions are all carried out from $t=0$ to $t=19$. See $[14,15]$ for more details.

In Fig. 3, we compare the Fourier spectra of the energy obtained by using the $2 / 3$ dealiasing method with those obtained by the Fourier smoothing method. For a fixed resolution $1024 \times 768 \times 2048$, we can see that the Fourier spectra obtained by the Fourier smoothing method retain more effective 
Fourier modes than those obtained by the $2 / 3$ dealiasing method. This can be seen by comparing the results with the corresponding computations using a higher resolution $1536 \times$ $1024 \times 3072$ (the solid lines). Moreover, the Fourier smoothing method does not give the spurious oscillations in the Fourier spectra. In comparison, the Fourier spectra obtained by the $2 / 3$ dealiasing method produce some spurious oscillations near the $2 / 3$ cut-off point. We would like to emphasize that our Fourier smoothing method conserves the total energy extremely well, at least up to six digits of accuracy. More studies including the convergence of the enstrophy spectra can be found in $[14,15]$.

It is worth emphasizing that a significant portion of those Fourier modes beyond the $2 / 3$ cut-off position are still accurate for the Fourier smoothing method. This portion of the Fourier modes that go beyond the $2 / 3$ cut-off point is about $12 \sim$ $15 \%$ of total number of modes in each dimension. For 3D problems, the total number of effective modes in the Fourier smoothing method is about $20 \%$ more than that in the $2 / 3$ dealiasing method. For our largest resolution, we have about 4.8 billions unknowns. An increase of $20 \%$ effective Fourier modes represents a very significant increase in the resolution for a large scale computation.

\section{Dynamics depletion of vortex stretching}

In this section, we present some convincing numerical evidences which show that there is a strong dynamic depletion of vortex stretching due to local geometric regularity of the vortex lines. We first present the result on the growth of the maximum velocity in time, see Fig. 7. The growth rate of the maximum velocity plays a critical role in the non-blowup criteria of Deng-Hou-Yu [8,9]. As we can see from Fig. 7, the maximum velocity remains bounded up to $t=19$. This is in contrast with the claim in [18] that the maximum velocity blows up like $O\left((T-t)^{-1 / 2}\right)$ with $T=18.7$. We note that the velocity field is smoother than the vorticity field. Thus it is easier to resolve the velocity field than the vorticity field. We observe an excellent agreement between the maximum velocity fields computed by the two largest resolutions. Since the velocity field is bounded, the first condition of Theorem 1 is satisfied by taking $A=0$. Furthermore, since both $\nabla \cdot \xi$ and $\kappa$ are bounded by $O\left((T-t)^{-1 / 2}\right)$ in the inner region of size $(T-t)^{1 / 2} \times(T-t)^{1 / 2} \times(T-t)$ [18], the second condition of Theorem 1 is satisfied with $B=1 / 2$ by taking a segment of the vortex line with length $(T-t)^{1 / 2}$ within this inner region. Thus Theorem 1 can be applied to our computation, which implies that the solution of the 3D Euler equations remains smooth at least up to $T=19$.

We also study the maximum vorticity as a function of time. The maximum vorticity is found to increase rapidly from the initial value of 0.669 to 23.46 at the final time $t=19$, a factor of 35 increase from its initial value. Our computations show no sign of finite time blowup of the 3D Euler equations up to $T=19$, beyond the singularity time predicted by Kerr. The maximum vorticity computed by resolution $1024 \times 768 \times 2048$ agrees very well with that computed by resolution $1536 \times$ $1024 \times 3072$ up to $t=17.5$. There is some mild disagreement

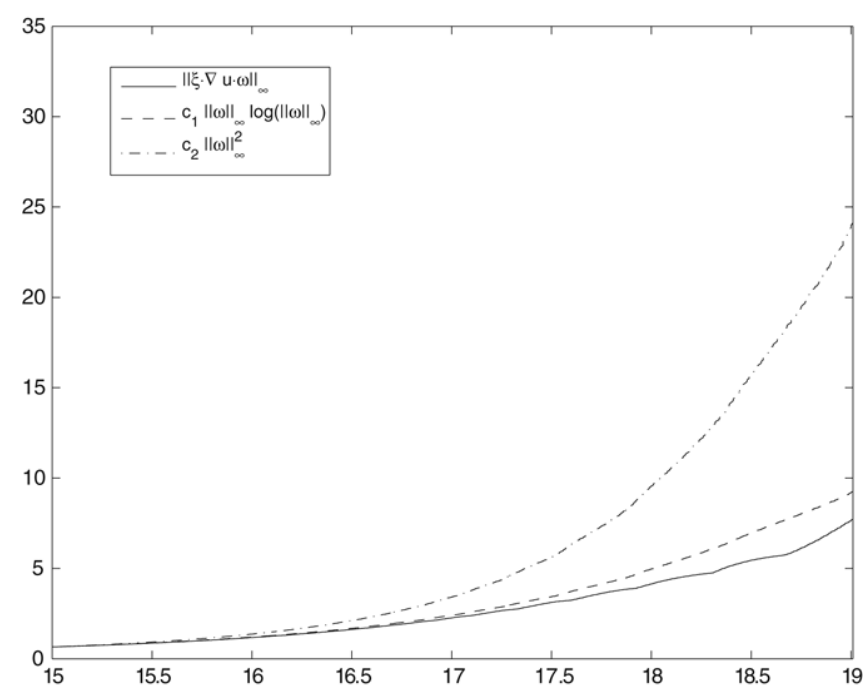

Fig. 8. Study of the vortex stretching term in time, resolution $1536 \times 1024 \times$ 3072. The fact $|\xi \cdot \nabla \mathbf{u} \cdot \boldsymbol{\omega}| \leq c_{1}|\boldsymbol{\omega}| \log |\boldsymbol{\omega}|$ plus $\frac{D}{D t}|\boldsymbol{\omega}|=\boldsymbol{\xi} \cdot \nabla \mathbf{u} \cdot \boldsymbol{\omega}$ implies $|\boldsymbol{\omega}|$ bounded by doubly exponential.

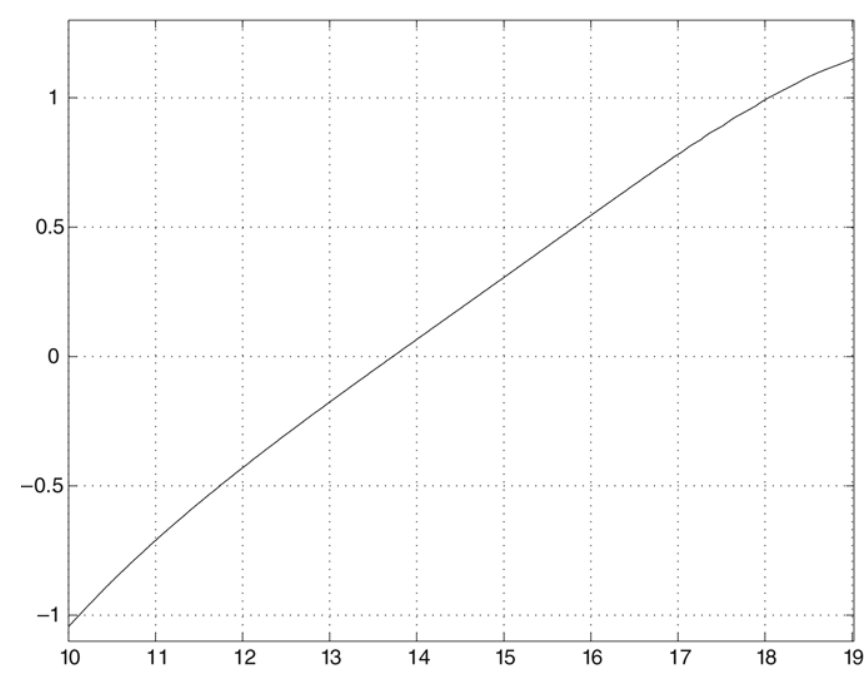

Fig. 9. The plot of $\log \log \|\omega\|_{\infty}$ vs time, resolution $1536 \times 1024 \times 3072$.

toward the end of the computation. This indicates that a very high space resolution is needed to capture the rapid growth of maximum vorticity at the final stage of the computation.

In order to understand the nature of the dynamic growth in vorticity, we examine the degree of nonlinearity in the vortex stretching term. In Fig. 8, we plot the quantity, $\|\xi \cdot \nabla \mathbf{u} \cdot \omega\|_{\infty}$, as a function of time. If the maximum vorticity indeed blew up like $O\left((T-t)^{-1}\right)$, as alleged in [17], this quantity should have been quadratic as a function of maximum vorticity. We find that there is tremendous cancellation in this vortex stretching term. It actually grows slower than $C\|\vec{\omega}\|_{\infty} \log \left(\|\vec{\omega}\|_{\infty}\right)$, see Fig. 8. It is easy to show that $\|\xi \cdot \nabla \mathbf{u} \cdot \boldsymbol{\omega}\|_{\infty} \leq C\|\vec{\omega}\|_{\infty} \log \left(\|\vec{\omega}\|_{\infty}\right)$ would imply at most doubly exponential growth in the maximum vorticity. Indeed, as demonstrated by Fig. 9, the maximum vorticity does not grow faster than doubly exponential in time. We have also generated the similar plot by extracting the data 


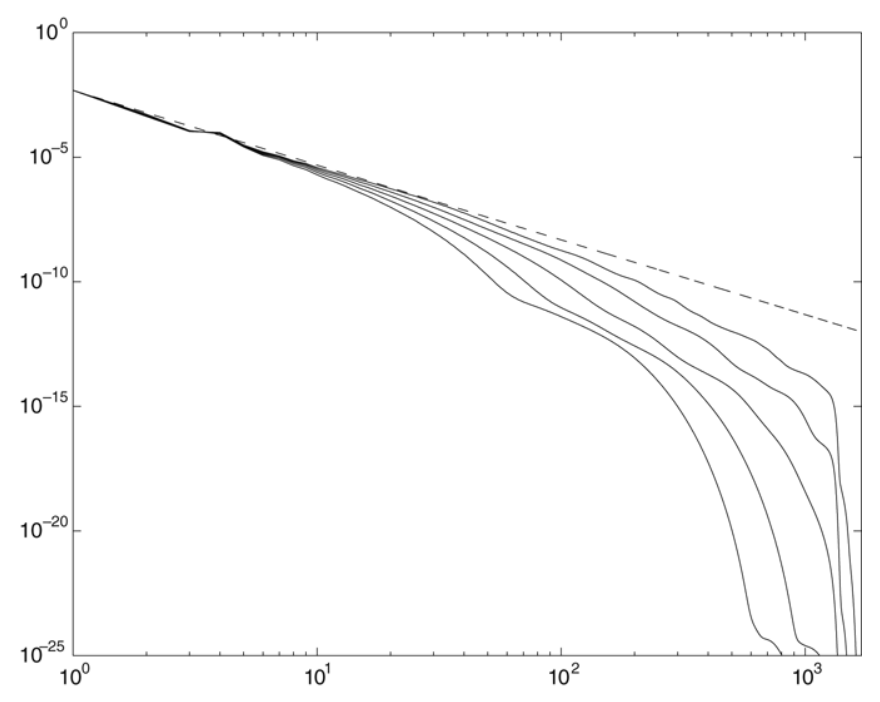

Fig. 10. The energy spectra for velocity at $t=15,16,17,18,19$ (from bottom to top) in log-log scale. The dashed line corresponds to $k^{-3}$.

from Kerr's paper [17]. We find that $\log \left(\log \left(\|\omega\|_{\infty}\right)\right)$ basically scales linearly with respect to $t$ from $14 \leq t \leq 17.5$ when his computations are still reasonably resolved. This implies that the maximum vorticity up to $t=17.5$ in Kerr's computations does not grow faster than doubly exponential in time. This is consistent with our conclusion.

We study the decay rate in the energy spectrum in Fig. 10 at $t=16,17,18,19$. A finite time blowup of enstrophy would imply that the energy spectrum decays no faster than $|k|^{-3}$. Our computations show that the energy spectrum approaches $|k|^{-3}$ for $|k| \leq 100$ as time increases to $t=19$. This is in qualitative agreement with Kerr's results. Note that there are only less than 100 modes available along the $\left|k_{x}\right|$ or $\left|k_{y}\right|$ direction in Kerr's computations, see Fig. 18 (a)-(b) of [17]. On the other hand, our computations show that the high frequency Fourier spectrum for $100 \leq|k| \leq 1300$ decays much faster than $|k|^{-3}$, as one can see from Fig. 10. This indicates that there is no blowup in enstrophy.

It is interesting to ask how the vorticity vector aligns with the eigenvectors of the deformation tensor. Recall that the vorticity equations can be written as [20]

$\frac{\partial}{\partial t} \boldsymbol{\omega}+(\mathbf{u} \cdot \nabla) \boldsymbol{\omega}=S \cdot \boldsymbol{\omega}, \quad S=\frac{1}{2}\left(\nabla \mathbf{u}+\nabla^{T} \mathbf{u}\right)$.

Let $\lambda_{1}<\lambda_{2}<\lambda_{3}$ be the three eigenvalues of $S$. The incompressibility condition implies that $\lambda_{1}+\lambda_{2}+\lambda_{3}=0$. If the vorticity vector aligns with the eigenvector corresponding to $\lambda_{3}$, which gives the maximum rate of stretching, then it is very likely that the 3D Euler equations would blow up in a finite time.

In Table 1, we document the alignment information of the vorticity vector around the point of maximum vorticity with resolution $1536 \times 1024 \times 3072$. In this table, $\theta_{i}$ is the angle between the $i$-th eigenvector of $S$ and the vorticity vector. One can see clearly that for $16 \leq t \leq 19$ the vorticity vector at the point of maximum vorticity is almost perfectly aligned with the second eigenvector of $S$. Note that the second eigenvalue, $\lambda_{2}$,
Table 1

The alignment of the vorticity vector and the eigenvectors of $S$ around the point of maximum vorticity with resolution $1536 \times 1024 \times 3072$

\begin{tabular}{llllllll}
\hline Time & $|\omega|$ & \multicolumn{1}{l}{$\lambda_{1}$} & $\theta_{1}$ & \multicolumn{1}{l}{$\lambda_{2}$} & $\theta_{2}$ & $\lambda_{3}$ & $\theta_{3}$ \\
\hline 16.012 & 5.628 & -1.508 & 89.992 & 0.206 & 0.007 & 1.302 & 89.998 \\
16.515 & 7.016 & -1.864 & 89.995 & 0.232 & 0.010 & 1.631 & 89.990 \\
17.013 & 8.910 & -2.322 & 89.998 & 0.254 & 0.006 & 2.066 & 89.993 \\
17.515 & 11.430 & -2.630 & 89.969 & 0.224 & 0.085 & 2.415 & 89.920 \\
18.011 & 14.890 & -3.625 & 89.969 & 0.257 & 0.036 & 3.378 & 89.979 \\
18.516 & 19.130 & -4.501 & 89.966 & 0.246 & 0.036 & 4.274 & 89.984 \\
19.014 & 23.590 & -5.477 & 89.966 & 0.247 & 0.034 & 5.258 & 89.994 \\
\hline
\end{tabular}

Here, $\theta_{i}$ is the angle between the $i$ th eigenvector of $S$ and the vorticity vector.

is positive and is about 20 times smaller in magnitude than the largest and the smallest eigenvalues. Although the alignment of the vorticity vector with the second eigenvector of the deformation tensor does not rule out a finite time blowup, this alignment is another indication that there is a strong dynamic depletion of vortex stretching.

\section{The Kida-Pelz high-symmetry data}

Another well-known numerical evidence for finite time Euler singularities is the Kida-Pelz high-symmetry initial data $[3,19]$. Some people have argued that the singular solution of the 3D Euler equations, if it exists, could be very unstable. A highly symmetric initial condition may have a better chance to produce a finite time singularity. It is also believed that a computer code needs to build in this symmetry property explicitly in order to capture the potentially unstable singular solution. This consideration motivated Boratav and Pelz to perform numerical simulations using a high-symmetry initial condition for the Navier-Stokes equations in [3].

The initial condition that Boratav and Pelz used [3] has the rotational symmetry and the permutation symmetry, which was first introduced by Kida [19]. Their simulations suggested a possible finite time blowup of the maximum vorticity in the limit of infinite Reynolds numbers. However, as they realized later, their simulations were under-resolved at later times when the solution became nearly singular. The vortex structure near the region of maximum vorticity motivated Pelz to construct a vortex filament model to understand this singular behavior. In [21], Pelz presented some numerical evidences which suggest that his filament model develop a self-similar blowup in a finite time. It is interesting to note that Pelz's self singular solution also falls into the critical case of the Deng-Hou-Yu local non-blowup criteria (see Theorem 2). To understand if the same initial condition that led to a finite time blowup in Pelz's filament model would lead to a finite time blowup of the full 3D Euler equations, we decide to repeat Pelz's computations.

Pelz's original filament model was designed for the entire free space. To perform the numerical simulation of the $3 \mathrm{D}$ Euler equations in the free space $R^{3}$ is very expensive. As a first step, we derive a corresponding periodic filament model. The periodic filament model involves an infinite sum over all the periodic images of the Biot-Savart kernel. This 


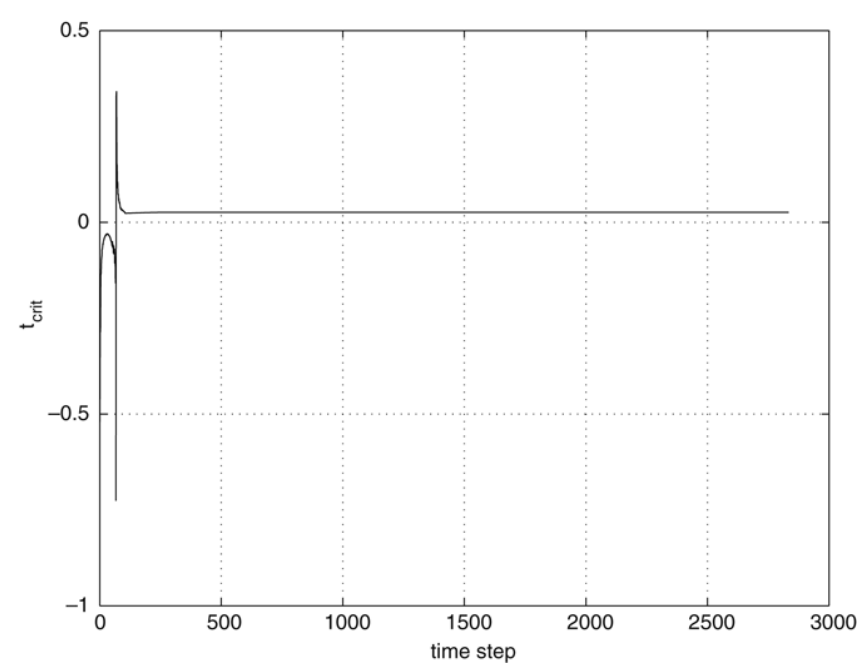

Fig. 11. The validity check of singularity fitting using the asymptotic expression $\|u\|_{\infty}=\frac{C}{\sqrt{t_{\text {crit }}-t}}$. The figure shows $t_{\text {crit }}$ as a function of the computational steps, with $t_{\text {crit }} \rightarrow 0.0257874$. Adaptive time stepping is used with the time step chosen to be proportional to the inverse of $\|u\|_{\infty}$.

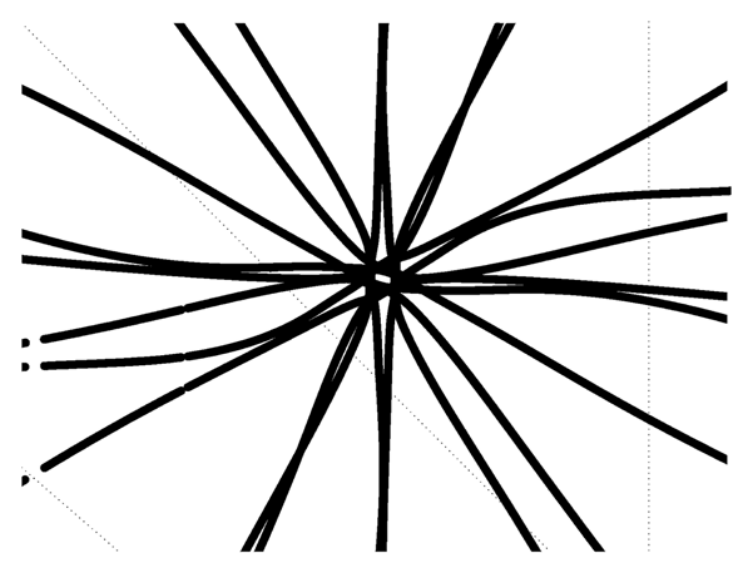

Fig. 12. The locations of the filaments at the end of our computation. The figure gives a closeup view of the filaments around the origin.

makes the computation of the periodic filament kernel more expensive than the one over the free space. To reduce the computational cost, we apply the Ewald summation formula, which significantly reduces the computational cost.

We solve the periodic filament model using an initial condition which is qualitatively the same as the one used by Pelz [21]. Our numerical computations show that the periodic filament model indeed develops a finite time selfsimilar singularity around $t=0.0257874$, see Figs. 11 and 12 . However, when we use the same initial condition to solve the full 3D Euler equations, we find that the solution of the 3D Euler equations has a completely different behavior from that of the filament model. We observe no finite time singularity for the 3D Euler equations using the same initial condition. We use a sequence of space resolutions with the two largest resolutions being $1024^{3}$ and $2048^{3}$. More than $100 \mathrm{~Gb}$ memory is used in our computation on the $2048^{3}$ computations. As we can see from Figs. 13 and 14, the growth of maximum vorticity

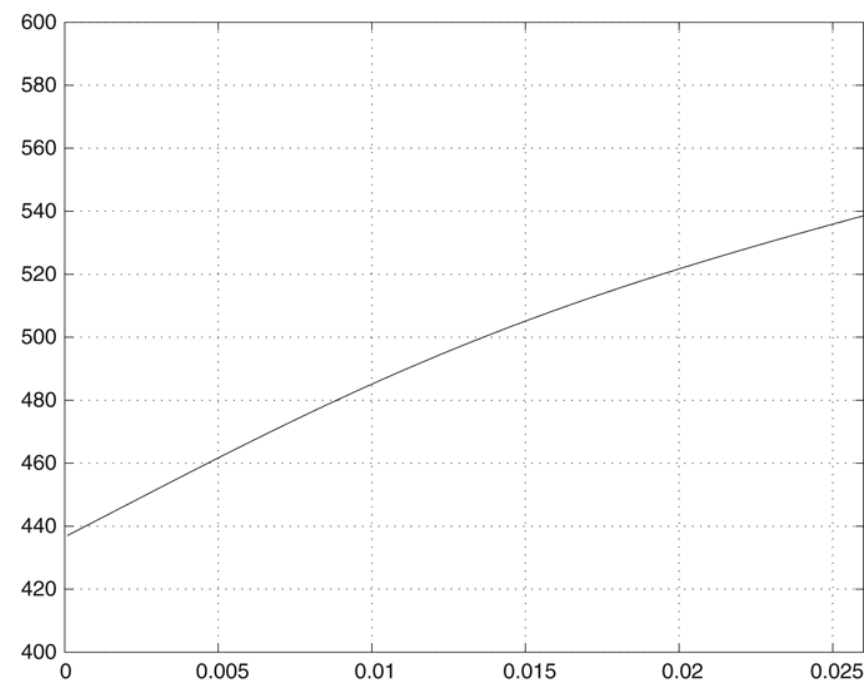

Fig. 13. Maximum vorticity in time of the full Euler equations with two resolutions: $1024^{3}$ (dashed line) vs $N=2048^{4}$ (solid line).

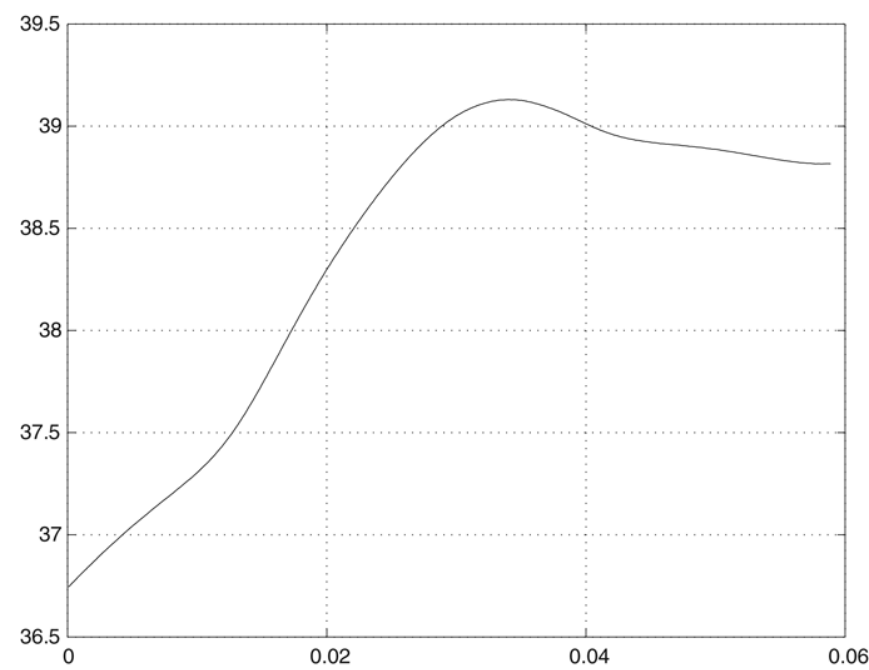

Fig. 14. Maximum velocity in time of the full Euler equations with resolution: $1024^{3}$. The maximum velocity seems to saturate at a later time.

in time is very mild. The maximum velocity is bounded and becomes saturated around $t=0.0325$. The 3D isosurface of the vortex tubes at $t=0.03$ plotted in Fig. 15 also shows that the vortex tubes remain quite regular. We remark that Grauer and his coworkers have recently carried out the full Euler simulation using a simplified Pelz's high-symmetry initial condition which consists of 12 straight parallel bars [13]. They find that the vortex tubes become severely flattened as they approach each other and the growth of maximum vorticity is only exponential in time.

Finally, we remark that we have repeated Boratav's and Pelz's Navier-Stokes computations [3] using the same initial condition, building both the rotational and permutation symmetries of the solution explicitly into our code. Our resolution study shows that their computations are resolved only up to $t=1.6$ when the growth of the maximum vorticity 


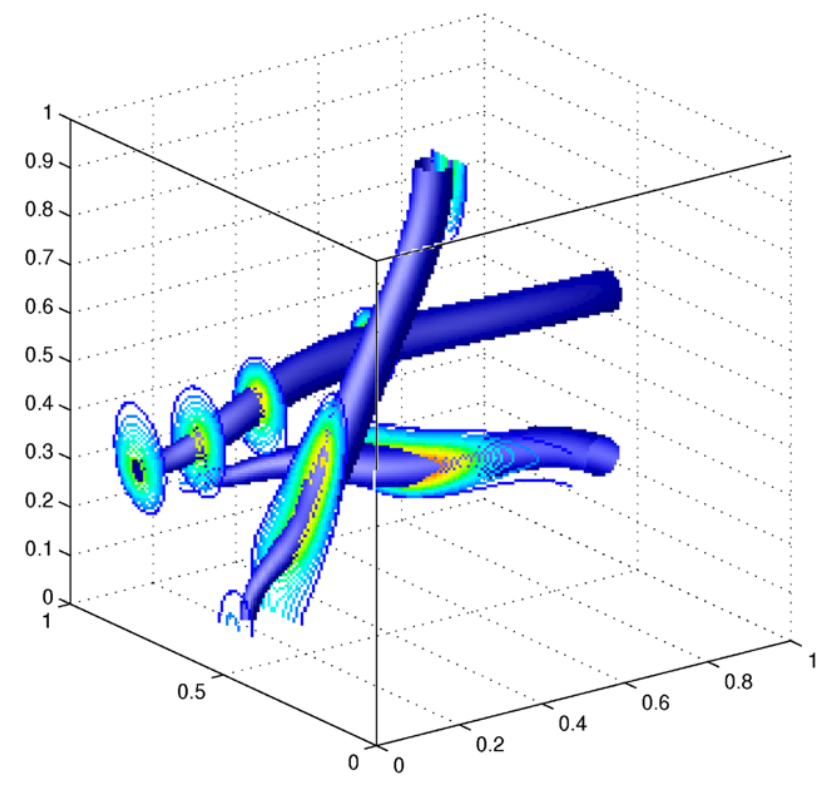

Fig. 15. The 50\% isosurface of $|\vec{\omega}|$ at $t=0.03$. Full 3D Euler equations.

is only exponential in time. The nearly singular growth of maximum vorticity around $t=2.06$ seems due to underresolution.

\section{Concluding remarks}

Our analysis and computations reveal a subtle dynamic depletion of vortex stretching. Sufficient numerical resolution is essential in capturing this dynamic depletion. Our computations for the two anti-parallel vortex tubes' initial data and the highsymmetry initial data show that the velocity is bounded and that the vortex stretching term is bounded by $C\|\omega\|_{L^{\infty}} \log \left(\|\omega\|_{L^{\infty}}\right)$. It is natural to ask if is this dynamic depletion generic? and what is the driving mechanism for this depletion of vortex stretching? Some exciting progress has been made recently in analyzing the dynamic depletion of vortex stretching and nonlinear stability for 3D axisymmetric flows with swirl [16]. The local geometric structure of the solution near the region of maximum vorticity and the anisotropic scaling of the support of maximum vorticity seem to play a key role in the dynamic depletion of vortex stretching.

\section{Acknowledgments}

We would like to thank Prof. Lin-Bo Zhang from the Institute of Computational Mathematics and the Center of High Performance Computing in Chinese Academy of Sciences for providing us with the computing resource to perform this large scale computational project. We also thank Prof. Robert Kerr for providing us with his Fortran subroutine that generates initial data. This work was supported in part by NSF under the NSF grants, FRG DMS-0353838, ITR ACI-0204932 and DMS-
0713670. Li was subsidized by the National Basic Research Program of China under the grant 2005CB321701.

\section{References}

[1] T.J. Beale, T. Kato, A.J. Majda, Remarks on the breakdown of smooth solutions of the 3-D Euler equations, Comm. Math. Phys. 96 (1984) 61-66.

[2] O.N. Boratav, R.B. Pelz, N.J. Zabusky, Reconnection in orthogonally interacting vortex tubes: Direct numerical simulations and quantifications, Phys. Fluids A 4 (1992) 581-605.

[3] O.N. Boratav, R.B. Pelz, Direct numerical simulation of transition to turbulence from a high-symmetry initial condition, Phys. Fluids 6 (1994) 2757-2784.

[4] M.E. Brachet, D.I. Meiron, S.A. Orszag, B.G. Nickel, R.H. Morf, U. Frisch, Small-scale structure of the Taylor-Green vortex, J. Fluid Mech. 130 (1983) 411.

[5] A. Chorin, The evolution of a turbulent vortex, Comm. Math. Phys. 83 (1982) 517.

[6] P. Constantin, Geometric statistics in turbulence, SIAM Rev. 36 (1994) 73.

[7] P. Constantin, C. Fefferman, A.J. Majda, Geometric constraints on potentially singular solutions for the 3-D Euler equation, Commun. PDEs 21 (1996) 559-571.

[8] J. Deng, T.Y. Hou, X. Yu, Geometric properties and non-blowup of 3-D incompressible Euler flow, Commun. PDEs 30 (2005) 225-243.

[9] J. Deng, T.Y. Hou, X. Yu, Improved geometric conditions for nonblowup of 3D incompressible Euler equation, Commun. PDEs 31 (2006) 293-306.

[10] J.D. Gibbon, The three-dimensional Euler equations: Where do we stand?, Physica D 237 (14-17) (2008) 1894-1970.

[11] R. Grauer, T. Sideris, Numerical computation of three dimensional incompressible ideal fluids with swirl, Phys. Rev. Lett. 67 (1991) 3511.

[12] R. Grauer, C. Marliani, K. Germaschewski, Adaptive mesh refinement for singular solutions of the incompressible Euler equations, Phys. Rev. Lett. 80 (1998) 19.

[13] T. Grafke, H. Homann, J. Dreher, R. Grauer, Numerical simulations of possible finite time singularities in the incompressible Euler equations: Comparison of numerical methods, Physica D 237 (14-17) (2008) 1932-1936.

[14] T.Y. Hou, R. Li, Dynamic depletion of vortex stretching and non-blowup of the 3-D incompressible Euler equations, J. Nonlinear Sci. 16 (2006) 639-664.

[15] T.Y. Hou, R. Li, Computing nearly singular solutions using pseudospectral methods, J. Comput. Phys. 226 (2007) 379-397.

[16] T.Y. Hou, C. Li, Dynamic stability of the 3D axisymmetric Navier-Stokes equations with swirl, Comm. Pure Appl. Math., published online on August 27, 2007 (doi:10.1002/cpa.20213).

[17] R.M. Kerr, Evidence for a singularity of the three dimensional, incompressible Euler equations, Phys. Fluids 5 (1993) 1725-1746.

[18] R.M. Kerr, Velocity and scaling of collapsing Euler vortices, Phys. Fluids 17 (2005) 075103.

[19] S. Kida, Three-dimensional periodic flows with high symmetry, J. Phys. Soc. Jpn. 54 (1985) 2132.

[20] A.J. Majda, A.L. Bertozzi, Vorticity and Incompressible Flow, Cambridge University Press, Cambridge, 2002.

[21] R.B. Pelz, Locally self-similar, finite-time collapse in a high-symmetry vortex filament model, Phys. Rev. E 55 (1997) 1617-1626.

[22] A. Pumir, E.E. Siggia, Collapsing solutions to the 3-D Euler equations, Phys. Fluids A 2 (1990) 220-241.

[23] M.J. Shelley, D.I. Meiron, S.A. Orszag, Dynamical aspects of vortex reconnection of perturbed anti-parallel vortex tubes, J. Fluid Mech. 246 (1993) 613. 